\title{
A Comparison of the Clinical and Radiographic Results of Press Fit Condylar Rotating-Platform High-Flexion and Low Contact Stress Mobile Bearing Prosthesis in Total Knee Arthroplasty: Short term Results
}

\author{
Shin Woo Nam, MD, Yong Seuk Lee, MD, PhD, Ji Hoon Kwak, MD, Nam Ki Kim, MD and Beom Koo Lee, \\ $\mathrm{MD}, \mathrm{PhD}$ \\ Department of Orthopedic Surgery, Gil Medical Center, Gachon University of Medicine and Science, Inchon, Korea
}

Purpose: This study compared the results of rotating-platform high-flexion (RP-F) total knee arthroplasty with low contact stress (LCS) for clinical and radiographical assessment after a short-term period.

Materials and Methods: 68 total knee arthroplasties using a RP-F and LCS system were analyzed retrospectively. Thirty-five of the 68 were osteoarthritic knees and were followed-up for more than 2 years. The clinical evaluation included range of motion (ROM), Knee Society Knee Score and Function Score (KSKS and KSFS), tailor position and kneeling. The radiographic evaluation included femorotibial angle, position of implants, radiolucent line and position of patella.

Results: The postoperative ROM, KSKS, and KSFS improved statistically in both implants. Comparing RP-F with LCS there were statistically no differences in ROM ( $p=0.863)$, KSKS ( $p=0.835)$, KSFS ( $p=0.535)$ and tailor position $(\mathrm{p}=0.489)$. There were no significant radiographic differences.

Conclusions: Total knee arthroplasty with RP-F and LCS showed similar clinical and radiographic results; it also showed excellent and predictable results at the short-term follow up. However, in RP-F there was 1 case of early osteolysis, 1 case of patella clunk syndrome and 1 case of painful patella crepitus; therefore, further case studies and follow-up are needed.

Key words: Knee, Osteoarthritis, Total knee arthroplasty, PFC Sigma RP-F, Low contact stress.

\section{Introduction}

Mobile-bearing knee prostheses were designed to increase the congruity of the articular surface and decrease polyethylene wear and component loosening after total knee arthroplasty (TKA) $)^{1,2}$.

Received June 9, 2011; Revised (1st) June 26, 2011;

(2nd) August 25, 2011; (3rd) September 20, 2011;

Accepted October 4, 2011.

Correspondence to: Beom Koo Lee, MD, PhD.

Department of Orthopedic Surgery, Gil Medical Center, Gachon University, 21 Namdong-daero 774 Beon-gil, Incheon 405-760, Korea. Tel: +82-32-460-3384, Fax: +82-32-468-5437

Email: bklee@gilhospital.com

This is an Open Access article distributed under the terms of the Creative Commons Attribution Non-Commercial License (http://creativecommons.org/licenses/by-nc/3.0/) which permits unrestricted non-commercial use, distribution, and reproduction in any medium, provided the original work is properly cited.
Restoration of flexion after TKA is crucial for successful clinical outcome and patient satisfaction especially in eastern countries where people require the use of high flexion positions including the tailor position and kneeling ${ }^{3)}$. Mobile-bearing high-flexion knee prostheses can be more effective in this regard than fixedbearing prostheses due to design characteristics and rotation of bearing during hyperflexion ${ }^{4,5)}$. In this study, we retrospectively compared the clinical and radiographic results of TKAs using the low contact stress (LCS; DePuy Inc., Warsaw, IN, USA) system and those using a high-flexion prosthesis, PFC Sigma RP-F (Press Fit Condylar, Sigma rotating-platform high-flexion, Johnson and Johnson Professional Inc., Raynham, MA, USA) system under the hypothesis that the mobile-bearing high-flexion knee prosthesis would result in more favorable clinical outcomes.

\section{Materials and Methods}

Between December 2006 and January 2010, 68 patients underwent TKA using either the RP-F or LCS system. Of them, 
35 patients with a minimum follow-up of 2 years were enrolled in this study. In the RP-F group, there were 16 patients (24 cases), all of whom were female. Their mean age was 67 years (range, 59 to 76 years) and the mean follow-up period was 39.9 months. In the LCS group, there were 19 patients (26 cases) comprising 1 male and 18 females. Their mean age was 68.8 years (range, 60 to 77 years) and the mean follow-up period was 25.6 months. The indication for TKA was degenerative arthritis in all patients.

The surgery was performed by the same surgeon using a medial parapatella approach in all patients. Cement was used for tibial and femoral fixation and the patella was not replaced. Tibial resection was followed by femoral resection and the posterior cruciate ligament was removed in all cases. The flexion/ extension gap was measured intraoperatively using a gapper to achieve mediolateral balance. Joint exercises started from the 1 st postoperative day using a continuous passive movement machine. From the 2nd postoperative day, partial weight-bearing using crutches or a walker was allowed. Full weight-bearing active exercises were permitted from the 7 th postoperative day.

Age, gender, body mass index (BMI), flexion contracture of the knee, further flexion, and genu varum angle were recorded preoperatively. For the evaluation of knee function, the Knee Society Knee Score (KSKS) and Knee Society Functional Score (KSFS) were assessed preoperatively and postoperatively ${ }^{6}$. In addition, the possibility of tailor position and kneeling and the presence of anterior crepitus and patellar clunk syndrome were examined. For radiographic assessment, radiologic component position and radiolucent line on the anteroposterior and lateral radiographs obtained at the last follow-up were evaluated according to the American Knee Society Roentgenographic Evaluation and Scoring System ${ }^{7}$. The following variables were measured on the radiographs to assess the component position:

Table 1. Comparison of Preoperative Variables between RP-F Group and LCS Group

\begin{tabular}{lcc}
\hline Preoperative variables & RP-F group & LCS group \\
\hline Age $(\mathrm{yr})$ & $67.0 \pm 7.5$ & $68.8 \pm 5.4$ \\
Body-mass index $(\mathrm{BMI})$ & $26.2 \pm 3.8$ & $27.8 \pm 3.4$ \\
Flexion contracture $\left(^{\circ}\right)$ & $5.2 \pm 8.6$ & $5.2 \pm 6.5$ \\
Further flexion $\left(^{\circ}\right)$ & $111.7 \pm 31.8$ & $112.7 \pm 27.6$ \\
Genu varum angle $\left(^{\circ}\right)$ & $0.6 \pm 6.1$ & $1.5 \pm 4.3$ \\
KSKS & $48.2 \pm 19.8$ & $40.2 \pm 22.0$ \\
KSFS & $42.9 \pm 25.7$ & $39.9 \pm 24.1$ \\
\hline
\end{tabular}

Values are presented as mean \pm standard deviation.

RP-F: rotating-platform high-flexion, LCS: low contact stress, KSKS: Knee Society Knee Score, KSFS: Knee Society Function Score. the medial angle between the femoral articular surface and the femoral axis $(\alpha)$ and the medial angle between the tibial implant and the tibial axis $(\beta)$ on the anteroposterior view and the acute angle between the line perpendicular to the femoral articular surface and the femoral axis $(\gamma)$ and the posterior angle between the tibial articular surface and the tibial axis $(\delta)$ on the lateral view. For the assessment of the femorotibialb (limb) alignment, the femorotibial angle defined as the acute angle between the femoral axis and the tibial axis was measured. The thickness of a radiolucent line was measured in units of $\mathrm{mm}$ and the added values were transformed into a score with $\leq 4$ considered insignificant, $\geq 5$ and $\leq 9$ requiring follow-up, and $\geq 10$ having a high probability of fixation failure regardless of the presence of clinical symptoms. The Insall-Salvati ratio $^{8)}$ on the lateral view and the congruence angle and tilt angle ${ }^{9)}$ on the Merchant view were measured.

Improvements in the range of motion (ROM), KSKS, and KSFS from the preoperative to the postoperative period were assessed using the Wilcoxon rank sum test. For comparison of the clinical results between the groups, the Mann-Whitney $\mathrm{U}$ test was used. The chi-square test was used to analyze the possibility of tailor position and kneeling and the radiolucent line score. Statistical significance was set at $\mathrm{p}<0.05$.

\section{Results}

\section{Clinical Results}

No statistically significant intergroup differences were observed in the preoperative variables including age, gender, BMI, flexion contracture, further flexion, genu varum angle, and KSKS and KSFS (Table 1).

The mean flexion contracture and further flexion were significantly improved between the preoperative and the last follow-up evaluations in both groups: from $5.2^{\circ}$ to $0.7^{\circ}$ $(\mathrm{p}=0.005)$ and from $111.7^{\circ}$ to $126.3^{\circ}(\mathrm{p}=0.046)$, respectively, in the RP-F group and from $5.2^{\circ}$ to $1.7^{\circ}(\mathrm{p}=0.026)$ and from $112.7^{\circ}$

Table 2. Clinical Results according to Range of Motion, KSKS and KSFS

\begin{tabular}{lcc}
\hline Postoperative variables & RP-F group & LCS group \\
\hline Flexion contracture $\left(^{\circ}\right)$ & $0.7 \pm 1.7$ & $1.7 \pm 3.2$ \\
Further flexion $\left(^{\circ}\right)$ & $126.3 \pm 10.3$ & $125.0 \pm 11.6$ \\
KSKS & $91.4 \pm 9.5$ & $92.2 \pm 11.5$ \\
KSFS & $87.8 \pm 11.4$ & $87.4 \pm 12.6$ \\
\hline
\end{tabular}

Values are presented as mean \pm standard deviation.

RP-F: rotating-platform high-flexion, LCS: low contact stress, KSKS: Knee Society Knee Score, KSFS: Knee Society Function Score. 
to $125^{\circ}$ ( $\mathrm{p}=0.027$ ), respectively, in the LCS group. However, no statistically significant intergroup differences were noted regarding the flexion contracture $(\mathrm{p}=0.149)$ and the further

Table 3. Results of Femorotiboal Angle, Radiologic Component Position and Patella Position

\begin{tabular}{lrr}
\hline Postoperative variables & RP-F group & LCS group \\
\hline Femorotibial angle $\left({ }^{\circ}\right)$ & Valgus $5.9 \pm 2.6$ & Valgus $5.8 \pm 3.6$ \\
$\beta\left(\left(^{\circ}\right)^{\text {a) }}\right.$ & $89.0 \pm 2.2$ & $89.6 \pm 1.6$ \\
$\Delta\left({ }^{\circ}\right)^{\text {b) }}$ & $85.9 \pm 4.1$ & $82.8 \pm 2.4$ \\
Insall-Salvati ratio & $1.15 \pm 0.18$ & $1.19 \pm 0.15$ \\
Congruence angle $\left(^{\circ}\right)$ & $-7.6 \pm 11.9$ & $-11.9 \pm 12.7$ \\
Tilt angle $\left({ }^{\circ}\right)$ & $5.1 \pm 2.7$ & $6.6 \pm 4.4$ \\
\hline
\end{tabular}

Values are presented as mean \pm standard deviation.

RP-F: rotating-platform high-flexion, LCS: low contact stress.

${ }^{a} \beta$ : acute angle between tibial implant and tibial axis, ${ }^{\text {b) }} \delta$ : posterior angle between tibial articular surface and tibial axis. flexion ( $\mathrm{p}=0.986)$ (Table 2).

The KSKS and KSFS improved significantly between the preoperative and the last follow-up evaluations in both groups: from 48.2 to $91.4(\mathrm{p}=0.000)$ and from 42.9 to $87.8(\mathrm{p}=0.000)$, respectively, in the RP-F group and from 40.2 to $92.2(\mathrm{p}=0.000)$ and from 39.9 to $87.4(\mathrm{p}=0.000)$, respectively, in the LCS group. However, no statistically significant intergroup differences were found regarding the KSKS $(\mathrm{p}=0.389)$ and KSFS $(\mathrm{p}=0.722)$ (Table 2).

Tailor position and kneeling was possible in 19 cases (79.2\%) and 6 cases (25\%), respectively, in the RP-F group and in 21 cases $(80.7 \%)$ and 4 cases (15.4\%), respectively, in the LCS group. However, no statistically significant intergroup differences were found $(\mathrm{p}=0.489)$.

\section{Radiographic Results}

The mean femorotibial angle was changed from a varus of $0.6^{\circ}$ to a valgus of $5.9^{\circ}$ in the RP-F group and from a valgus of $1.5^{\circ}$ to
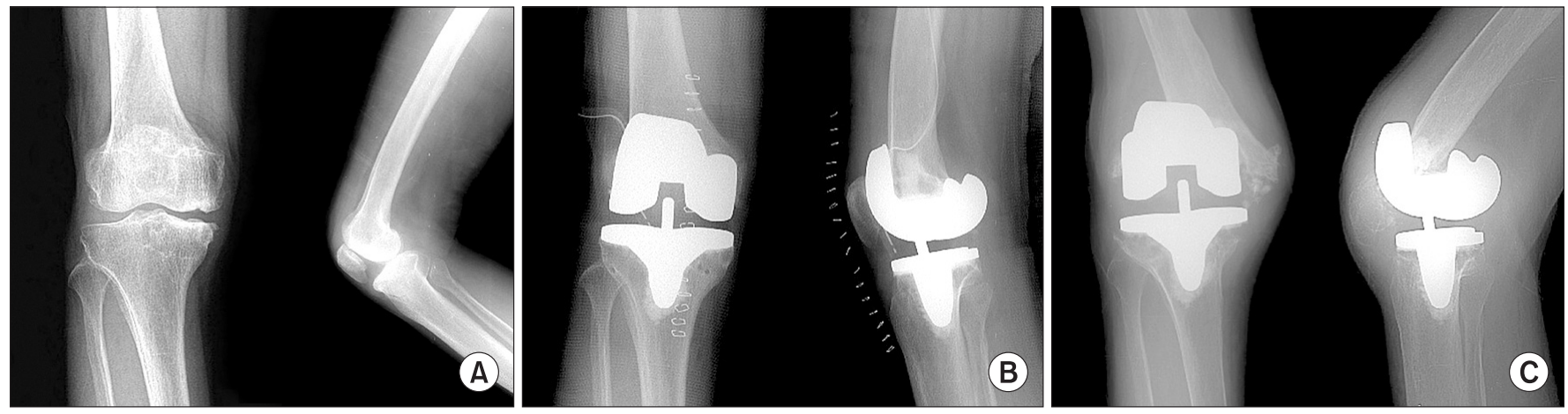

Fig. 1. (A) Preoperative radiographs of a 71-year-old female show degenerative osteoarthritis. (B) Postoperative radiographs show a total knee replacement status using the rotating-platform high-flexion system. (C) At postoperative 27 months, radiographs show severe osteolysis on femoral component.

A

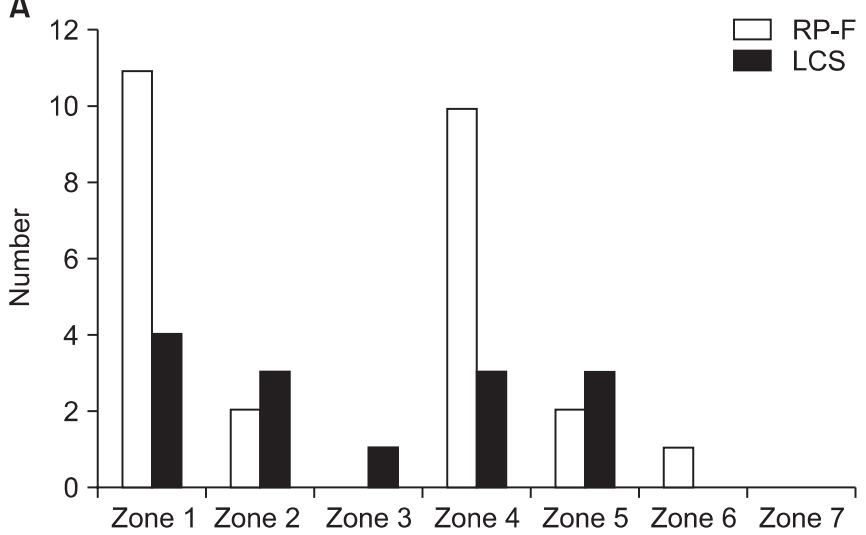

B

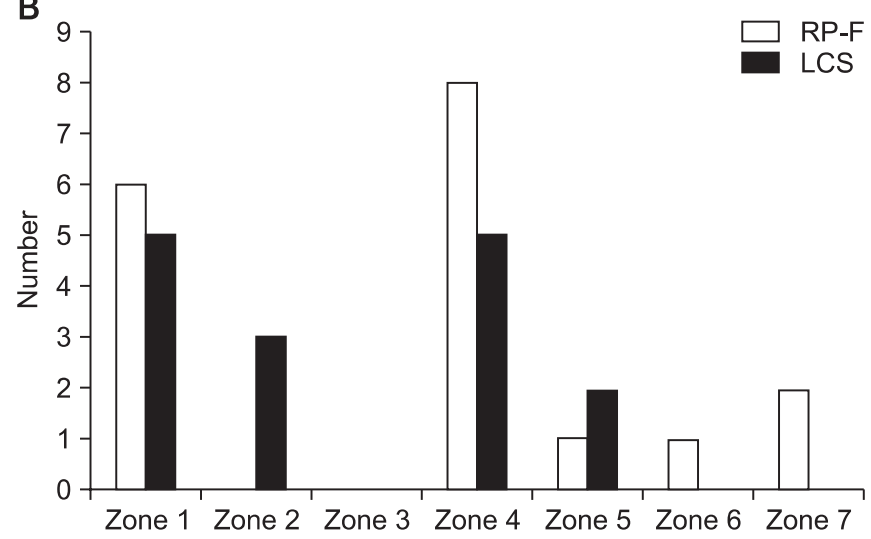

Fig. 2. (A) The incidence of radiolucent line in the anterior-posterior view of tibia. (B) The incidence of radiolicent line in lateral view of femur. RP-F: rotating-platform high-flexion, LCS: low contact stress. 

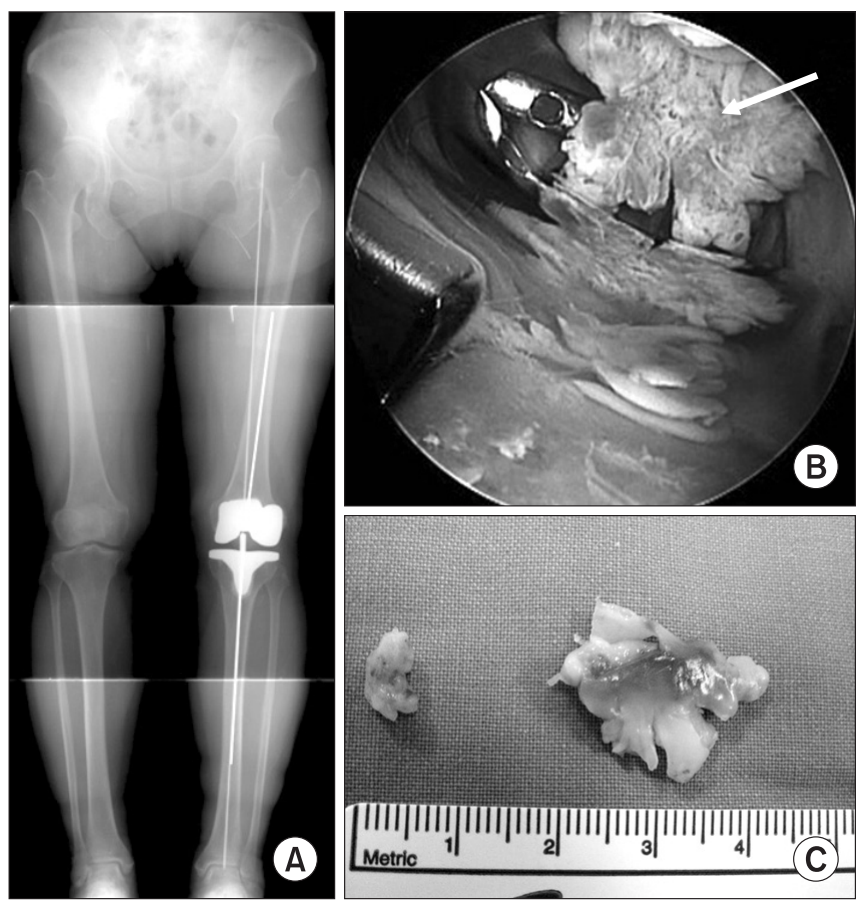

Fig. 3. (A) Postoperative radiograph of 73-year-old female shows good alignment. Femorotibial angle 5.6 $6^{\circ}$ (B) The arrow indicate the presence of nodules at the intercondylar notch. (C) Two $\mathrm{cm}$-sized and $0.5 \mathrm{~cm}$ sized nodules were removed.

a valgus of $5.8^{\circ}$ in the LCS group. Regarding the implant location, the mean $\beta$ value was $89.0^{\circ}$ in the RP-F group and $89.6^{\circ}$ in the LCS group and the mean $\delta$ value was $85.9^{\circ}$ in the RP-F group and $82.8^{\circ}$ in the LCS group (Table 3 ).

In the RP-F group, in all cases except for 1 case with severe osteolysis and early loosening that required a revision surgery (Fig. 1), the radiolucent line score was $\leq 4$ and most of the radiolucent lines were observed in the posterior femoral condyle (zone 4 ) in 7 cases (29.2\%). In the LCS group, the score was $\leq 4$ in all cases except for a score of 5 in 1 case, and a $1 \mathrm{~mm}$ radiolucent line was observed in the posterior femoral condyle in 4 cases (15.4\%), but no statistical significance was found ( $\mathrm{p}=0.134)$ (Fig. 2).

The mean Insall-Salvati ratio, congruence angle, and tilt angle were $1.15,-7.6$, and $5.1^{\circ}$, respectively, in the RP-F group and 1.19 , $-11.9^{\circ}$, and $6.6^{\circ}$, respectively, in the LCS group. There was no statistically significant intergroup difference in the Insall-Salvati ratio $(\mathrm{p}=0.515)$ and congruence angle $(\mathrm{p}=0.059)$, except for the tilt angle $(\mathrm{p}=0.026)$ (Table 3$)$.

\section{Complications}

In the RP-F group, early loosening that required a revision arthroplasty was observed in 1 case (Fig. 1) and painful patella crepitus was observed in 2 cases (8.3\%). Of the latter 2 cases, patellar clunk syndrome was observed in 1 case where pain and crepitus disappeared and ROM increased after arthroscopic nodule excision (Fig. 3).

\section{Discussion}

Conventional fixed-bearing knee prostheses have been associated with component wear and loosening ${ }^{1,2}$. However, mobile-bearing prostheses can be advantageous in reducing wear and loosening theoretically because they are specifically designed to improve congruity and decrease stresses to the knee such as rotational stresses ${ }^{10-14)}$. In addition, the femoral component design that alter the radius of femoral component allows for greater flexion, which eventually results in increased ROM. The LCS system, that has a wide contact surface include reduced contact stress and constrained force and increased ROM due to the various radii of curvature of the femoral component ${ }^{1,15,16}$. The PFC Sigma RP-F system, that reduces the radius of curvature of the posterior femoral condyles, increasing posterior femoral rollback with a post-cam mechanism ${ }^{17}$. In addition, the increase in posterior condylar offset and rotation of the bearing surface improves internal rotation of the tibia for high flexion of the knee.

However, we could not find significant differences between the groups regarding the clinical scores and maximum ROM during flexion in this study. According to Ritter ${ }^{18)}$, the preoperative flexion range is a major determinant of the postoperative further flexion. In this study, we could not find notable differences in the preoperative flexion range between the groups. Kurosaka et al. ${ }^{19)}$ reported that patients with a weak extensor mechanism due to long-standing arthritis could have a reduced range of flexion under weight-bearing movement. All the patients in our study had degenerative arthritis and were advanced in age with a mean of 68.1 years. Therefore, we considered that the postoperative ROM was more related to the preoperative ROM than the prosthesis design.

Femoral component loosening after TKA can be influenced by infection, implant design, malalignment, bone strength, cemented fixation technique, and excessive use of the knee joint. Factors that can cause osteolysis and bone loss include operative technique, patient characteristics, and implant design ${ }^{20-24)}$. The compressive force applied to the posterior femoral condyles with the knee at $90^{\circ}$ of flexion is three times greater than body weight ${ }^{17}$. Based on the observation of 15 cases of femoral component loosening 
after 1,600 total knee arthroplasties, King and Scott ${ }^{25)}$ reported that when support for the implant is inadequate, the compressive force on the posterior femoral condyles is transmitted in the form of a shear force at the distal interface with the implant and lead to a tensile force at the anterior interface, and accordingly the distal posterior interface acts as a pivot point for component loosening. Han et al. ${ }^{26)}$ encountered 27 cases (38\%) of femoral component loosening in 72 cases of total knee replacements and attributed this to extensive removal of the posterior femoral condyles that results in inadequate support and excessive movement of the femoral component in high flexion ${ }^{26)}$. Kim et al. ${ }^{27)}$ could not find significant differences in radiographic results and survival rate in a comparison study of two mobile-bearing prostheses including a high-flexion design. However, early loosening and osteolysis requiring a revision surgery was observed in 1 case in the RP-F group and radiolucent lines were observed in the posterior femoral condyles (zone 4) in 7 cases (29.2\%) in the RP-F group compared to 5 cases (15.4\%) in the LCS group in this study. There were no differences between the groups regarding the factors that could influence the occurrence of osteolysis including diagnosis, infection, alignment, and ROM. The surgery was performed by the same surgeon in all cases. In addition, we took care to inject cement in the dough stage in both groups and perform drilling in sclerotic bone lesions. Therefore, we believed that osteolysis 1) progresses from the posterior femoral condyles as King and Scott ${ }^{25)}$ reported, 2) can be caused by extensive posterior femoral condyle resection because the incidence was higher in the RP-F group where more resection was unavoidable due to the implant design compared to the LCS group and 3) can be influenced by stresses that are focused on the posterior femoral condyles and post because of activities requiring high degrees of flexion especially for people engaged in agriculture ${ }^{28,29)}$.

In our opinion, early loosening can be prevented with the use of a stem or lug in addition to the RP-F implant, as suggested by King and $\mathrm{Scott}^{25)}$, and Insall and Scott ${ }^{30)}$, or proper cementing on the posterior condyle.

Patella clunk syndrome was first described by Hozack et al. ${ }^{31)}$ and similar complications of TKA were referred to as tethered patellar syndrome by Thorpe et al. ${ }^{8}$ and synovial entrapment syndrome by Pollock et al. ${ }^{32}$. Although authors used different terms, these syndromes belong to the same disease entity: they occur when a fibrous nodule grows excessively and induce impingement between the component. The cause of this syndrome reported that posterior stabilized prostheses, sharp anterior edge of the femoral component, and narrow shape and anterior position of the $\mathrm{cam}^{8,33,34)}$. Other possible causative factors include patella height, thickness, and abnormal patella tracking ${ }^{8,31,33,35)}$.

In this study, there were 2 cases of painful patella crepitus in the RP-F group and 1 of these was patellar clunk syndrome that was treated with arthroscopic resection. There were no factors that could affect the postoperative soft tissue and ligament balancing including infection, proximal tibial correction osteotomy, and revision surgery. Considering that the alignment and implant location were within the normal range, the patellar clunk syndrome can be attributable to the design characteristics of the RP-F implant that has box-cutting and is extended to the anterior aspect of the proximal femur, and has an edge that curves sharply anterior to the intercondylar notch ${ }^{27,33,34,36)}$. No statistically significant difference was found in the congruence angle. The LCS group appeared to have better patella tracking compared to the RP-F group because the lateral tilt was significantly smaller in the former group.

\section{Conclusions}

The short-term clinical and radiographic results of TKA did not show significant differences between the PFC Sigma RP-F system and the rotating platform LCS system. Regarding the complications, severe osteolysis and early loosening was observed in 1 case in the RP-F group and patella clunk syndrome was suspected in 2 more cases in the RP-F group compared to the LCS group. We believe that further studies involving a larger number of patients and a longer follow-up period are necessary.

\section{References}

1. Argenson JN, Scuderi GR, Komistek RD, Scott WN, Kelly MA, Aubaniac JM. In vivo kinematic evaluation and design considerations related to high flexion in total knee arthroplasty. J Biomech. 2005;38:277-84.

2. Buechel FF, Pappas MJ. New Jersey low contact stress knee replacement system. Ten-year evaluation of meniscal bearings. Orthop Clin North Am. 1989;20:147-77.

3. Ritter MA, Campbell ED. Effect of range of motion on the success of a total knee arthroplasty. J Arthroplasty. 1987;2:957.

4. Kim TH, Lee DH, Bin SI. The NexGen LPS-flex to the knee prosthesis at a minimum of three years. J Bone Joint Surg Br. 2008;90:1304-10.

5. Nakamura E, Banks SA, Tanaka A, Sei A, Mizuta H. Threedimensional tibiofemoral kinematics during deep flexion 
kneeling in a mobile-bearing total knee arthroplasty. J Arthroplasty. 2009;24:1120-4.

6. Insall JN, Dorr LD, Scott RD, Scott WN. Rationale of the Knee Society clinical rating system. Clin Orthop Relat Res. 1989;(248):13-4.

7. Ewald FC. The Knee Society total knee arthroplasty roentgenographic evaluation and scoring system. Clin Orthop Relat Res. 1989;(248):9-12.

8. Thorpe CD, Bocell JR, Tullos HS. Intra-articular fibrous bands. Patellar complications after total knee replacement. J Bone Joint Surg Am. 1990;72:811-4.

9. Merchant AC, Mercer RL, Jacobsen RH, Cool CR. Roentgenographic analysis of patellofemoral congruence. J Bone Joint Surg Am. 1974;56:1391-6.

10. Marrs H, Barton DC, Jones RA, Ward IM, Fisher J, Doyle C. Comparative wear under four different tribological conditions of acetylene enhanced cross-linked ultra high molecular weight polyethylene. J Mater Sci Mater Med. 1999;10:333-42.

11. McEwen HM, Barnett PI, Bell CJ, Farrar R, Auger DD, Stone MH, Fisher J. The influence of design, materials and kinematics on the in vitro wear of total knee replacements. J Biomech. 2005;38:357-65.

12. Menchetti PP, Walker PS. Mechanical evaluation of mobile bearing knees. Am J Knee Surg. 1997;10:73-81.

13. O'Connor JJ, Goodfellow JW. Theory and practice of meniscal knee replacement: designing against wear. Proc Inst Mech Eng H. 1996;210:217-22.

14. Weaver JK, Derkash RS, Greenwald AS. Difficulties with bearing dislocation and breakage using a movable bearing total knee replacement system. Clin Orthop Relat Res. 1993;(290):244-52.

15. Ezzet KA, Garcia R, Barrack RL. Effect of component fixation method on osteolysis in total knee arthroplasty. Clin Orthop Relat Res. 1995;(321):86-91.

16. Goodfellow JW, O'Connor J. Clinical results of the Oxford knee. Surface arthroplasty of the tibiofemoral joint with a meniscal bearing prosthesis. Clin Orthop Relat Res. 1986;(205):21-42.

17. Morrison JB. The mechanics of the knee joint in relation to normal walking. J Biomech. 1970;3:51-61.

18. Ritter MA. High-flexion knee designs: more hype than hope? In the affirmative. J Arthroplasty. 2006;21:40-1.

19. Kurosaka M, Yoshiya S, Mizuno K, Yamamoto T. Maximizing flexion after total knee arthroplasty: the need and the pitfalls. J Arthroplasty. 2002;17:59-62.
20. Bae DK, Chung SW, Lee CW, Yang HS. Osteolysis around screw in cementless total knee replacement. J Korean Orthop Assoc. 1998 33:1301-6.

21. Cadambi A, Engh GA, Dwyer KA, Vinh TN. Osteolysis of the distal femur after total knee arthroplasty. J Arthroplasty. 1994;9:579-94.

22. Ries MD, Guiney W Jr, Lynch F. Osteolysis associated with cemented total knee arthroplasty. A case report. J Arthroplasty. 1994;9:555-8.

23. Schmalzried TP, Callaghan JJ. Wear in total hip and knee replacements. J Bone Joint Surg Am. 1999;81:115-36.

24. Whiteside LA. Effect of porous-coating configuration on tibial osteolysis after total knee arthroplasty. Clin Orthop Relat Res. 1995;(321):92-7.

25. King TV, Scott RD. Femoral component loosening in total knee arthroplasty. Clin Orthop Relat Res. 1985;(194):285-90.

26. Han HS, Kang SB, Yoon KS. High incidence of loosening of the femoral component in legacy posterior stabilised-flex total knee replacement. J Bone Joint Surg Br. 2007;89:145761.

27. Kim YH, Kim JS, Park JW, Joo JH. Comparison of the low contact stress and press fit condylar rotating-platform mobile-bearing prostheses in total knee arthroplasty: a prospective randomized study. J Bone Joint Surg Am. 2011;93:1001-7.

28. Cho SD, Youm YS, Park KB. Three- to six-year followup results after high-flexion total knee arthroplasty: can we allow passive deep knee bending? Knee Surg Sports Traumatol Arthrosc. 2011;19:899-903.

29. Nakayama K, Matsuda S, Miura H, Iwamoto Y, Higaki H, Otsuka K. Contact stress at the post-cam mechanism in posterior-stabilised total knee arthroplasty. J Bone Joint Surg Br. 2005;87:483-8.

30. Insall JN, Scott WN. Surgery of the knee. 3rd ed. Newyork: Churchill Livingstone; 2001. p1663-4.

31. Hozack WJ, Rothman RH, Booth RE Jr, Balderston RA. The patellar clunk syndrome. A complication of posterior stabilized total knee arthroplasty. Clin Orthop Relat Res. 1989;(241):203-8.

32. Pollock DC, Ammeen DJ, Engh GA. Synovial entrapment: a complication of posterior stabilized total knee arthroplasty. J Bone Joint Surg Am. 2002;84:2174-8.

33. Beight JL, Yao B, Hozack WJ, Hearn SL, Booth RE Jr. The patellar "clunk" syndrome after posterior stabilized total knee arthroplasty. Clin Orthop Relat Res. 1994;(299):139-42.

34. Chiu KY, Ng TP, Tang WM, Yau WP. Review article: knee 
flexion after total knee arthroplasty. J Orthop Surg (Hong Kong). 2002;10:194-202.

35. Diduch DR, Scuderi GR, Scott WN, Insall JN, Kelly MA. The efficacy of arthroscopy following total knee replacement.
Arthroscopy. 1997;13:166-71.

36. Yau WP, Wong JW, Chiu KY, Ng TP, Tang WM. Patellar clunk syndrome after posterior stabilized total knee arthroplasty. J Arthroplasty. 2003;18:1023-8. 